\title{
Eruptive Xanthoma During Antiretroviral Therapy
}

\author{
Olushola L. Akinshemoyin Vaughn ${ }^{1}$, Kenneth B. Gordon ${ }^{1}$, J. Njeri Wainaina ${ }^{2}$ \\ ${ }^{1}$ Department of Dermatology, Medical College of Wisconsin, Milwaukee, WI, USA \\ ${ }^{2}$ Department of Medicine, Division of Infectious Disease, Medical College of Wisconsin, Milwaukee, WI, USA
}

\begin{abstract}
We present a case of eruptive xanthoma in a man living with HIV after changing antiretroviral therapy. People living with HIV are at increased risk of dyslipidemia due to the infection itself and the use of antiretroviral medications. Prompt recognition of eruptive xanthomas may allow for timely diagnosis of metabolic disorders and dyslipidemia in patients being treated for HIV infection. J Microbiol Infect Dis 2019; 9(1): 43-45.
\end{abstract}

Keywords: Eruptive xanthoma, hypertriglyceridemia, metabolic syndrome, HIV, dolutegravir

\section{INTRODUCTION}

Eruptive xanthomas are a benign deposition of lipids in the dermis reported in about $10 \%$ of patients with severe hypertriglyceridemia [1]. It is a clinical diagnosis, based on the appearance of numerous monomorphic yellow-orange papules in a characteristic extensor distribution on the extremities, buttocks, and hands. Risk factors include obesity, diabetes mellitus, alcohol abuse, and certain systemic medications. Several classes of antiretroviral medication have been associated with lipodystrophy, most classically protease inhibitors (PIs).

While a medication-induced etiology of eruptive xanthoma may be suspected based on time course, causality is often impossible to verify. Nonetheless, prompt recognition of eruptive xanthomas in this population may help guide antiretroviral therapy. In all cases, timely diagnosis of eruptive xanthoma may reduce risk of acute pancreatitis and coronary artery disease. We present a case of a man living with HIV for illustration of the clinical presentation, evaluation, and natural history of cutaneous disease.

\section{CASE}

A 41-year-old man was evaluated for a 2-week history of a papular rash on his forearms, thighs, and buttocks (Figure 1). He had a history of anxiety, gastroesophageal reflux, hyperlipidemia, and HIV infection for which he was treated with aripiprazole, duloxetine, clonazepam, omeprazole, simvastatin, and coformulated efavirenz, emtricitabine, and tenofovir. HIV infection was well-controlled with sustained virological suppression and good immune recovery as evidenced by an absolute CD4 count of $1078 / \mu \mathrm{L}$. Due to increasing anxiety, his antiretroviral therapy was changed to dolutegravir, tenofovir alafenamide, and emtricitabine as efavirenz can exacerbate psychiatric symptoms including anxiety. He noticed a rash four days after the medication change and contacted his infectious disease provider who instructed him to hold the dolutegravir as it was his newest medication. He was unable to go in for an appointment but sent photographs of the rash via the patient portal. The rash did not look like a typical drug eruption so after a week, he was instructed to resume dolutegravir. The rash persisted and he presented to his primary care provider who prescribed doxycycline for 7 days. With no improvement in the eruption, the patient was referred to the dermatology service for consultation.

Findings on evaluation by dermatology were striking for numerous monomorphic firm yelloworange $2-5 \mathrm{~mm}$ papules symmetrically on the extensor forearms, thighs, and buttocks. There were no periocular or tendinous lesions. When

Correspondence: Dr. Olushola Akinshemoyin Vaughn, M.D., Department of Dermatology, Medical College of Wisconsin, Milwaukee, WI, USA 
asked directly, he admitted to polyuria, polydipsia, and dizziness. He also reported having started a low-carbohydrate diet six weeks earlier after routine testing showed his glucose was elevated.

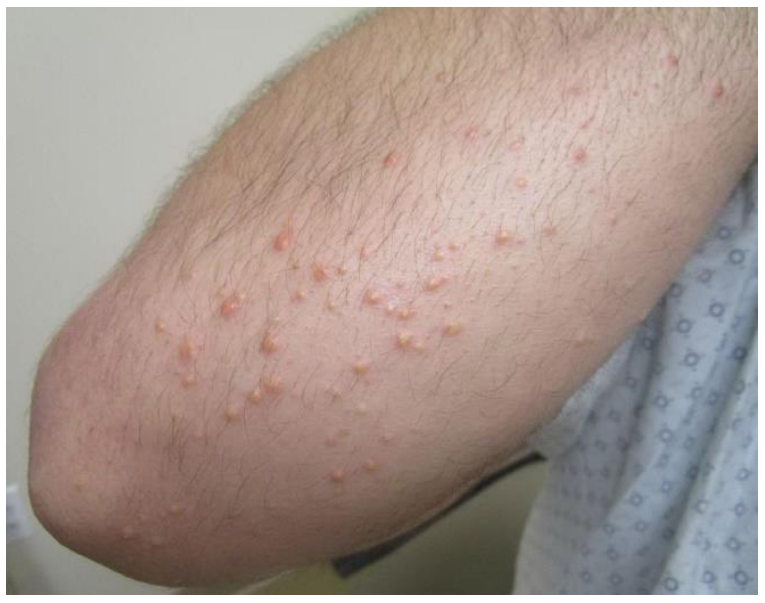

Figure 1. Numerous monomorphic firm yellow-orange $2-5 \mathrm{~mm}$ papules on the right extensor forearm.

A lipid panel was ordered but could not be run because it was grossly hemolyzed and lipemic. A fasting specimen drawn the following morning could not be quantified for the same reason. He was evaluated in the Emergency Department and subsequently hospitalized for management of malignant hyperlipidemia and diabetic ketoacidosis. Admission bloodwork was most notable for triglyceride level of $3513 \mathrm{mg} / \mathrm{dL}$ and $\mathrm{HbA} 1 \mathrm{c}$ of $14.2 \%$. He was treated with diabetic ketoacidosis protocol and was discharged on insulin, fenofibrate, atorvastatin, and fish oil. Antiretroviral therapy was ultimately adjusted at infectious disease follow up to raltegravir, emtricitabine, and tenofovir. At 1-year follow-up he had complete resolution of xanthomas and good virologic control.

\section{DISCUSSION}

Eruptive xanthomas are a benign deposition of lipids in the dermis and are highly suggestive of hypertriglyceridemia. They are associated with elevation of other lipid fractions including very low-density lipoproteins and chylomicrons, and can also be the initial sign of diabetes. They are retrospectively reported in about $10 \%$ of patients with severe hypertriglyceridemia, usually at levels exceeding $3000 \mathrm{mg} / \mathrm{dL}$. The appearance of eruptive xanthomas should prompt triglyceride measurement especially in patients with newly diagnosed or poorly controlled diabetes, due to the risk of acute pancreatitis and coronary artery disease. Eruptive xanthomas can be diagnosed clinically; biopsy is not required and was not performed in our case. Gradual resolution of cutaneous lesions is typically observed with triglyceride normalization [1]

People living with HIV are at increased risk of dyslipidemia due to the infection, the use of antiretroviral medications, and the longer life expectancy that is the outcome of effective control. Lipid alterations directly related to HIV infection are well characterized in people living with HIV [2]. This is likely related to the fact that enveloped viruses, including HIV-1, use cholesterol for their envelope structure and infectivity [3]. In addition, some antiretroviral medication classes are associated with various dyslipidemias. Lipodystrophy is classically associated with protease inhibitors (PIs), and more recently with nucleoside reverse transcriptase inhibitors (NRTIs) such as zidovudine and non-nucleoside reverse transcriptase inhibitors (NNRTIs) such as efavirenz. The mechanism of antiretroviralassociated dyslipidemia is not fully understood but may be due to structural similarity between regions of the HIV-1 protease and two human proteins involved in lipid metabolism [2].

Dolutegravir is an integrase strand transfer inhibitor (InSTI) approved in 2013 [4]. It is a preferred first-line agent and is used in combination with dual nucleoside reverse transcriptase inhibitors. A 2015 randomized controlled trial found dolutegravir to be lipid neutral compared to efavirenz, raltegravir, and ritonavir-boosted darunavir [5]. The original drug trials, however, reported a rate of hyperglycemia at $10 \%$ and a statistically significant increase in mean cholesterol, though incidence was not reported [4].

In conclusion, InSTIs, similarly to PIs, NRTIs, and NNRTIs, may induce severe metabolic adverse effects including insulin resistance and dyslipidemia. Prompt recognition of eruptive xanthomas, which are usually associated with severe hypertriglyceridemia, may allow for timely diagnosis of metabolic disorders and 
dyslipidemia in patients being treated for HIV infection with antiretroviral medications.

\section{ACKNOWLEDGMENTS}

\section{Financial disclosures:}

Akinshemoyin Vaughn and J. Njeri Wainaina: None reported.

Kenneth B. Gordon: KBG has received honoraria for serving as a consultant and/or grants as an investigator from AbbVie, Almirall, Amgen, Boehringer Ingelheim, Bristol-Myers Squibb, Celgene, Dermira, Eli Lilly, GlaxoSmithKline, Janssen, Leo Pharma, Novartis, Pfizer, Regeneron, Sanofi-Aventis, Sun, and UCB.

Declaration of Conflicting Interests: The authors declare that they have no conflict of interest.

Funding source: The authors received no third party funding for this study.

\section{REFERENCES}

1. Kashif $M$, Kumar $H$, Khaja $M$. An Unusual Presentation of Eruptive Xanthoma: A Case Report and Literature Review. Ed. Sergio Gonzalez Bombardiere. Medicine 2016; 95(37): e4866.

2. da Cunha J, Maselli LM, Stern AC, Spada C, Bydlowski SP. Impact of antiretroviral therapy on lipid metabolism of human immunodeficiency virus-infected patients: Old and new drugs. World J Virol 2015; 4(2): 56-77.

3. Campbell SM, Crowe SM, Mak J. Virionassociated cholesterol is critical for the maintenance of HIV-1 structure and infectivity. AIDS 2002; 16(17):2253-2261.

4. Tivicay Dolutegravir (as dolutegravir sodium). Laval, Quebec, Canada: ViiV Healthcare ULC; February 2017.

5. Quercia R, Roberts J, Martin-Carpenter L, Zala C. Comparative Changes of Lipid Levels in Treatment-Naive, HIV-1-Infected Adults Treated with Dolutegravir vs. Efavirenz, Raltegravir, and Ritonavir-Boosted Darunavir-Based Regimens Over 48 Weeks. Clin Drug Investig 2015; 35(3): 211-219. 